\title{
HIV disclosure in the workplace
}

\author{
S. Degroote ${ }^{1,2}$, D. Vogelaers ${ }^{1,3}$, R. Koeck ${ }^{4}$, R. Borms ${ }^{4}$, L. De Meulemeester ${ }^{5}$, \\ D. Vandijck ${ }^{1,2,6}$
}

${ }^{1}$ Department of General Internal Medicine, Ghent University Hospital, Ghent, Belgium, ${ }^{2}$ Department of Public Health, Faculty of Medicine and Health Sciences, Ghent University, Ghent, Belgium, ${ }^{3}$ Department of Internal Medicine, Faculty of Medicine and Health Sciences, Ghent University, Ghent, Belgium, ${ }^{2}$ Sensoa vzw, Flemish Expertise Centre for Sexual Health, Antwerp, Belgium, ${ }^{5}$ Faculty of Medicine and Health Sciences, Ghent University, Ghent, Belgium, ${ }^{6}$ Department of Health Economics and Patient Safety, Faculty of Business Economics, Hasselt University, Diepenbeek, Belgium

Objectives: As HIV is currently a chronic and manageable disease, an increasing amount of people living with HIV (PLHIV) are (again) active on the labour market. Since research on this topic is scarce, this study aimed to explore experiences of PLHIV in the workplace, especially concerning disclosure and adherence to antiretroviral therapy.

Methods: A questionnaire was developed and validated in collaboration with Sensoa (Flemish expertise centre for sexual health) and participants were recruited using flyers and announcements on websites.

Results: A total of 54 PLHIV completed the questionnaire, among whom 50 (92.6\%) males. Half of the participants did not disclose their HIV status in the workplace, mostly due to being afraid of social or professional consequences. Those who disclosed, reported no changes in the workplace or even reported receiving more empathy. A minority of participants have to take antiretroviral medication at work and they reported no particular problems related to medication intake.

Conclusion: Despite improved solidarity and information campaigns, many PLHIV still do not disclose their HIV status in the workplace, most frequently due to fear for discrimination. More actions are warranted, as well as addressing possible self-stigma. Adherence to antiretroviral therapy in the workplace posed little or no problems.

Keywords: HIV, Disclosure, Work

\section{Introduction}

Current treatment strategies have turned HIV infection into a chronic disease and life expectancy for people living with HIV (PLHIV) continues to increase. ${ }^{1-3}$ Moreover, treatment options are still expanding and new antiretroviral agents are less toxic and more convenient than earlier antiretroviral therapy (ART). As such, the vast majority of PLHIV can currently lead a relatively normal life. Consequently, PLHIV can make a professional career or may return to work after a period of disability. ${ }^{4}$

Previous research showed that a majority of PLHIV work. Furthermore, employment seemed to positively influence health-related quality of life. ${ }^{5}$ The present study aimed to explore experiences of PLHIV in the workplace concerning disclosure and adherence to ART.

Correspondence to: S. Degroote, Department of General Internal Medicine, Ghent University Hospital, De Pintelaan 185, De Pintepark, 9000 Ghent, Belgium. Email: sophiem.degroote@ugent.be

\section{Materials and Methods}

In collaboration with Sensoa, the Flemish expertise centre for sexual health, a research project was conceived. Literature research was performed and emerging themes were discussed together with the experts of Sensoa, resulting in a scientific and practice-based approach.

\section{Participants}

All PLHIV who were currently working or had been working in the past, were eligible for inclusion. The target audience was reached by flyers distributed in the Flemish AIDS reference centres in Antwerp, Brussels and Ghent and during HIV cafés (informal meetings among peers), by announcements on the website of Sensoa, their daughter website 'www.levenmethiv.be' (= 'living with hiv'), their Facebook account and the community website 'HivMix'.

\section{Questionnaire}

Results from the literature research were used to outline the structure of the questionnaire. ${ }^{4,6-9}$ In consultation with Sensoa, the questions were chosen and formulated, resulting in a tree-structured questionnaire examining 
two main themes: disclosure and medication-intake in the workplace. In addition, socio-demographic data (gender, age), date of HIV diagnosis and information about the work situation (work schedule, job type, etc.) were also included. It was decided to use an online questionnaire as this is an easy, low-cost and quick method to collect data.

\section{Statistical analyses}

All results were exported from the questionnaire software ('Qualtrics') to SPSS.22 (SPSS, Inc., Chicago, IL, USA). Descriptive analyses were performed and are reported via numbers and percentages. Associations were examined via chi-square tests.

\section{Results}

Population

A total of 67 PLHIV participated in the study, among whom 54 fully completed the questionnaire. There were 50 men and four women, with a median age of 42 years (IQR 33-51 years). Forty-four people were currently working (12 blue-collar workers ${ }^{*}, 25$ whitecollar workers*, five self-employed and two public servants). Ten people were unemployed and one of them did not return to work after HIV diagnosis, rendering other questions no longer applicable. Almost three quarters of the remaining participants worked full-time (39/53, 73.6\%).

\section{Disclosure}

Almost half of the respondents did not inform any of the colleagues about his/her seropositive status (23/ 53, 43.4\%). People who disclosed, mostly told close colleagues only $(22 / 53,41.5 \%)$, an official trustee (13/ $53,24.5 \%)$ or executives $(12 / 53,22.6 \%)$. When being asked what hindered disclosure, half of the people reported to have fear for professional consequences (such as discharge, loss of clients) $(28 / 53,52.8 \%)$ and to be afraid for social consequences (such as discrimination, nagging) $(26 / 53,49.1 \%)$. Twenty-one participants $(42.0 \%)$ worried about people telling others and 11 respondents $(22.0 \%)$ mentioned to have followed someone's advice to not disclose at the workplace.

Those who disclosed, mostly did this of their own free will $(23 / 30,76.7 \%)$. Justifying their changing health state $(10 / 30,33.3 \%)$ or the (more frequent) absences $(8 / 30,26.7 \%)$, but also the desire to be honest and not to live with a secret were the most cited reasons to disclose $(4 / 30,13.3 \%)$.

In the majority of the cases, no professional changes appeared after disclosure $(18 / 30,60.0 \%)$, or the change was positive (i.e. more understanding from colleagues, 8/30, 26.7\%). Nonetheless, three

* Blue collar workers perform manual labour. White collar workers perform professional, managerial or administrative labour. participants $(10 \%)$ declare that they got fired after they had disclosed.

As compared to blue-collar workers, significantly more white-collar workers had disclosed their seropositive status in the workplace $(4 / 12,33.3 \%$ vs $17 /$ $25,68.0 \%, P=0.046)$. Disclosure was not found to be associated with other factors (gender, age, time since diagnosis, disclosure to environment, $P>0.05$ ).

\section{Medication intake}

Forty-seven participants $(88.7 \%)$ reported to take ART, among whom $38(80.9 \%)$ once daily. Twentyone participants took their medication in the workplace and none of them reported problems doing so, neither towards colleagues, neither towards infrastructure. Half of the 26 others took the decision not to take medication at work consciously, in consultation with their physician. Variable work time tables were most frequently reported as a hindering factor to adherence $(5 / 21,23.8 \%)$. On the other hand, access to food $(8 / 21,38.1 \%)$, drinks $(7 / 21,33.3 \%)$ and the proficiency to quickly take ART $(7 / 21,33.3 \%)$ were reported to be supporting factors.

\section{Discussion}

Our findings confirm the general good health among PLHIV in Belgium. ${ }^{10}$ For this study population, being seropositive does not necessarily hinder a professional career, since the vast majority of respondents work full-time. Strikingly, almost half of the participants did not disclose in the workplace, despite the many solidarity campaigns, etc. This number is almost double as compared to French PLHIV $(29.9 \%){ }^{7}$ but less than British PLHIV $(61.5 \%){ }^{6}$ The lower disclosure percentage seen in the French study may be partly due to the fact that this study took place in 2002-2003. Ten years ago, openness about HIV may have been less frequent. The higher disclosure percentage in the UK study is probably biased by the study population, almost completely consisting of homosexual, white men. They are more likely to disclose to employers than heterosexual men/women and homosexual men from ethnic minority groups. ${ }^{11}$

Fear for discrimination or discharge is high, and seems to be the main reason to not disclose. Previous research among French-speaking Belgian PLHIV showed that $6.5 \%$ of the people who disclosed experienced difficulties afterwards (being refused a job, blocked career, etc.) ${ }^{12}$ The latter demonstrates that information and solidarity campaigns from organizations as Sensoa are highly needed. Internalized stigma, the fear and shame PLHIV experience from within, remains an increasingly recognized additional barrier. ${ }^{13}$ Some PLHIV argue that disclosure will not give any added value, or they consider it a private affair. ${ }^{6}$ Hence, it should be acknowledged that the decision to 
disclose is a personal choice and that achieving 100\% disclosure might be an unrealistic goal.

Differences in job context may influence the decision to disclose the status. We have found that white-collar workers disclose more frequently than blue-collar workers. However, this issue should be further explored in bigger study samples and confounding factors should be taken into account. Those could be: type of working environment, the reputation of the person, colleagues' knowledge about HIV, anticipated negative consequences and awareness of the sexual orientation of the person living with HIV (mainly applicable for homosexual people). ${ }^{6,8}$

Data about medication intake in the workplace, revealed that this is less an issue than expected. Many PLHIV do not take ART at work, since certain regimens need to be taken before sleep or along with the main course, which is mostly in the evening. If they do take ART at work, they do not seem to encounter difficulties. Availability of accommodations, such as flexible work hours or easy access to food and drinks, were reported to be helpful, corresponding with previous research about adherence in the workplace. ${ }^{9}$

Some limitations of this study should be acknowledged. Our relatively small sample size does not fully represent the Belgian HIV cohort, since there are more female PLHIV (37 vs 7\%) and a lower percentage is on ART (75 vs 87\%). ${ }^{10}$ Our study methodology is also susceptible for recruitment bias towards people who are currently working. Moreover, our results should be further studied in depth by means of qualitative research (e.g. focus groups). Nevertheless, these first results already show tendencies and can inspire further actions and campaigns.

In conclusion, half of the PLHIV did not disclose their seropositive status in the workplace mainly due to fear for discrimination. The potential barriers need to be further explored.

\section{Acknowledgements}

The authors thank $\mathrm{Mr}$ Peter Vermeir for his assistance in this collaborative study.

\section{References}

1 Antiretroviral Therapy Cohort C. Life expectancy of individuals on combination antiretroviral therapy in high-income countries: a collaborative analysis of 14 cohort studies. Lancet. 2008;372(9635):293-9.

2 Bor J, Herbst AJ, Newell ML, Barnighausen T. Increases in adult life expectancy in rural South Africa: valuing the scale-up of HIV treatment. Science. 2013;339(6122):961-5.

3 May M, Gompels M, Delpech V, Porter K, Post F, Johnson M, et al. Impact of late diagnosis and treatment on life expectancy in people with HIV-1: UK Collaborative HIV Cohort (UK CHIC) Study. BMJ. 2011;343:d6016.

4 McGoldrick C. HIV and employment. Occup Med. 2012;62(4): 242-53.

5 Degroote S, Vogelaers DP, Vermeir P, Mariman A, De Rick A, Van Der Gucht B, et al. Socio-economic, behavioural, (neuro)psychological and clinical determinants of HRQoL in people living with HIV in Belgium: a pilot study. J Int AIDS Soc. $2013 ; 16(1): 18643$.

6 Douglas N. I just get on with it... A study of the employment experiences of gay and bisexual men and black African men and women living with HIV in the UK. London: NAT National AIDS Trust; 2009.

7 Dray-Spira R, Lert F, Group VS. Living and working with HIV in France in 2003: results from the ANRS-EN12-VESPA Study. AIDS. 2007;21 Suppl 1:S29-36.

8 Fesko SL. Disclosure of HIV status in the workplace: considerations and strategies. Health Soc Work. 2001;26(4): 235-44.

9 Torres-Madriz G, Lerner D, Ruthazer R, Rogers WH, Wilson IB. Work-related barriers and facilitators to antiretroviral therapy adherence in persons living with HIV infection. AIDS Behav. 2011;15(7):1475-82.

10 Van Beckhoven D, Buve A, Ruelle J, Seyler L, Sasse A, Belgian H. I. V. Cohort Study Group. A national cohort of HIVinfected patients in Belgium: design and main characteristics. Acta Clin Belg. 2012;67(5):333-7.

11 Elford J, Ibrahim F, Bukutu C, Anderson J. Disclosure of HIV status: the role of ethnicity among people living with HIV in London. J Acquir Immune Defic Syndr. 2008;47(4):514-21.

12 Pezeril C, Plateforme Prévention SIDA, l'Observatoire du sida et des sexualités. Enquête sur les conditions de vie des personnes vivant avec le VIH en Belgique francophone (Wallonie et Bruxelles). Brussels: Plateforme Prévention SIDA, l'Observatoire du sida et des sexualité; 2012.

13 Lee RS, Kochman A, Sikkema KJ. Internalized stigma among people living with HIV-AIDS. AIDS Behav. 2002;6(4):309-19. 